\title{
Characterisation of optical properties of solar nanofluids by an inverse problem based on a numerical model
}

\author{
Josep Forner-Escrig ${ }^{1}$, Alexandra Gimeno-Furió ${ }^{1}$, Roberto Palma ${ }^{2}$, Leonor Hernández ${ }^{1}$ and Rosa Mondragón ${ }^{1 *}$ \\ ${ }^{1}$ Department of Mechanical Engineering and Construction, Universitat Jaume I, Av. de Vicent Sos Baynat, s/n 12071 Castelló de la \\ Plana, Spain \\ ${ }^{2}$ Department of Structural Mechanics and Hydraulic Engineering, University of Granada, Campus Universitario Fuentenueva, Edf. \\ Politécnico, 18071 Granada, Spain
}

\begin{abstract}
Some nanoparticles (NPs) possess an outstanding photothermal conversion under optical illumination. For this reason, these NPs are under research in a wide variety of light-induced heating applications such as solar nanofluids, which could be used for direct light absorption in solar collectors. Experimental characterisation of solar nanofluids for their application to light-to-heat conversion processes requires a considerable amount of resources to determine the properties of this mixture, at the nanoscale level. On this ground, an inverse problem based on a high-frequency and light-to-heat finite element model is proposed in the present work to numerically predict the optical properties of these nanofluids. In particular, a cost function based on a L2 norm is formulated to compare experimental measurements and numerical predictions. Then, this function is minimised by means of heuristic techniques -specifically, genetic algorithms- and the desired properties can be determined. In conclusion, the current work presents a numerical tool that could help in the characterisation of properties of solar nanofluids and contribute to reduce the number of experiments to be conducted for this purpose.
\end{abstract}

\footnotetext{
* Corresponding author: mondragn@uji.es
} 


\section{Nomenclature}

\begin{tabular}{|c|c|c|}
\hline Quantity & Symbol & SI Unit \\
\hline $\begin{array}{c}\text { Electrical } \\
\text { conductivity }\end{array}$ & $\gamma$ & $1 /(\Omega \cdot \mathrm{m})$ \\
\hline $\begin{array}{c}\text { Excitation } \\
\text { pulsation }\end{array}$ & $\omega$ & $\mathrm{rad} / \mathrm{s}$ \\
\hline $\begin{array}{c}\text { Vacuum } \\
\text { permittivity }\end{array}$ & $\varepsilon_{0}$ & $\mathrm{~F} / \mathrm{m}$ \\
\hline $\begin{array}{c}\text { Imaginary part } \\
\text { of complex } \\
\text { relative } \\
\text { permittivity }\end{array}$ & $\varepsilon^{\prime \prime}$ & - \\
\hline $\begin{array}{c}\text { Wave amplitude } \\
\text { of the } \\
\text { sinusoidal } \\
\text { electric field }\end{array}$ & $\mathrm{E}_{0}$ & $\mathrm{~V} / \mathrm{m}$ \\
\hline
\end{tabular}

\section{Introduction}

The consumption of energy is continuously increasing worldwide since nowadays human lifestyle permanently demands energy to ensure the current life standards. Energy generation has traditionally relied on fossil fuels such as carbon and its derived products. However, the main disadvantage of this conventional energy source is that its availability is limited in time and it is responsible for environmental problems like global warming and air pollution.

In order to overcome this issue, renewable energies are at the focus of the scientific and technical communities with the aim of producing clean energy. One of the renewable energies under research is the solar one since two hours of irradiation could provide enough energy to cover the energetic needs in a year by 2035 [1].

Direct absorption solar collector (DASC) concept is an alternative to enhance the thermal efficiency of solar harvesting with respect to conventional solar collectors [2]. DASC allows solar energy to be volumetrically absorbed and transported by the working fluid itself. Therefore, the efficiency of DASC relies on the absorption capability of the fluid media. Since most of the heat transfer fluids (HTFs) are transparent, its absorption efficiency can be improved by adding nanoparticles (NPs) in suspension to the HTF.

The dispersion of nano-sized solids within a base fluid receives the name of nanofluid [3] and a specific branch of nanofluids, known as solar nanofluids [4,5] is currently under research since low concentrations of NPs allow significant improvements in solar energy harvesting. Different nanoparticles are tested for these solar thermal absorption systems and materials such as gold $(\mathrm{Au})$ are of special interest. Indeed, Au NPs possess remarkable photothermal conversion efficiency around their surface plasmon resonance, which occurs in the visible light spectrum. This part of light spectrum stands for $40 \%$ of the total solar energy and is often ineffectively absorded by transparent HTFs [6].
Therefore, mastering the optical properties of NPs is of key importance since they play a direct influence on the light absorption of solar nanofluids. However, bulk optical properties can be easily found in databases while material parameters at the nanoscale are harder to obtain. Since the experimental characterisation of optical properties of NPs is difficult to achieve due to the reduced size of the particles to be characterised and owing to the dispersion in the already existing measurements of optical properties, a numerical tool is proposed in this work in order to help in their characterisation.

The present numerical tool is based on the solution of an inverse problem, which consists in calculating the causal factors (or material properties in the present case) that produce a set of experimental measurements. The solution of an inverse problem depends on the minimisation of a cost function (CF) measuring the discrepancy between experimental and numerical results. These numerical results (forward problem) are obtained in the present work through the evaluation of a high-frequency and light-to-heat Finite Element (FE) model, which predicts the thermal increase undergone by the nanofluid when optically excited [7]. Then, the $\mathrm{CF}$ is minimised by making use of heuristic techniques of optimisation, namely, genetic algorithms (GA).

With regard to the experimental results for the formulation of the inverse problem, these were directly measured in a laboratory by making use of the experimental setup described in the work of GimenoFurió et al. [8]. To sum up, this experimental setup (see Figure 1) consists of a quartz glass tube in which the nanofluids were placed and which was irradiated by an artificial sunlight simulator. The evolution of temperature of the nanofluids was registered by means of a data acquisition system and measured by a K-type thermocouple. Another thermocouple was also used to measure the ambient temperature and the tests were performed in an isolated room to perform the experiments under more stable and reproducible conditions.

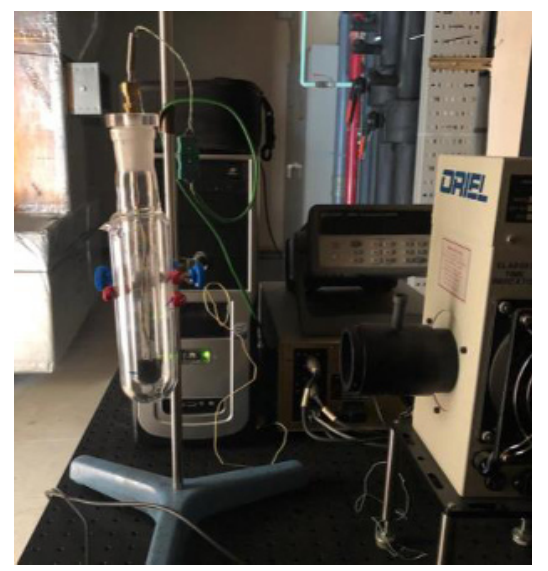

Fig. 1. Experimental setup for the measurement of temperature increase of optically excited nanofluids. 
In the present work, the numerical tool is applied to determine the optical properties (namely, imaginary part of relative permittivity and electrical conductivity) of water-based nanofluids with $\mathrm{Au}$ NPs dispersed in it and for different sizes of NPs within the base fluid.

\section{Analysis and modelling}

The proposed problem consists in the characterisation of optical properties of NPs, which can be stated as the calculation of these properties by means of an inverse problem.

The numerical tool selected for determining the response of the model presented in this work makes use of a light-to-heat FE model to predict the temperature increase undergone by the nanofluids (forward problem).

The governing equation of this problem is:

$$
\rho c \dot{\mathrm{T}}=-\underline{\nabla} \cdot \underline{\mathrm{q}}+\dot{\mathrm{S}},
$$

where the heat source term is computed as:

$$
\dot{\mathrm{S}}=\frac{1}{2} \cdot\left[\frac{\gamma}{\omega \varepsilon_{0}}+\varepsilon^{\prime \prime}\right] \omega \varepsilon^{\prime \prime} \mathrm{E}_{0}^{2},
$$

and the heat flux vector $\underline{\mathrm{q}}$ is defined by Fourier's law:

$$
\underline{\mathrm{q}}=\underline{-} \underline{\kappa} \cdot \underline{\nabla} \mathrm{T} .
$$

8-node hexahedral linear elements are used to solve the present model, which has been implemented in the research code FEAP [9], belonging to the University of California at Berkeley (USA), where the unknown (or degree of freedom) is the temperature.

The $\mathrm{CF}$, often referred to as objective function, $\mathrm{f}$ is defined as the quadratic difference between the numerical results $\psi_{\mathrm{i}}{ }^{\mathrm{NUM}}$ and the experimental measurements $\psi_{\mathrm{i}}^{\text {EXP }}$ :

$$
\mathrm{f}=\frac{1}{2 \mathrm{~N}} \sum_{\mathrm{i}=1}^{\mathrm{N}}\left(\psi_{\mathrm{i}}^{\mathrm{EXP}}-\psi_{\mathrm{i}}^{\mathrm{NUM}}\right)^{2}
$$
points.

where $\mathrm{N}$ represents the number of measuring

When the minimisation is performed by GA or other heuristic optimisation techniques, the CF is often regularised as $\mathrm{f}_{\mathrm{L}}$ :

$$
f_{L}=\log (f+\varepsilon),
$$

where $\varepsilon$ is a small unitless value (here considered as $\varepsilon=10^{-16}$ ) to guarantee the existence of $f_{L}$ when $f$ tends progressively towards zero [10].

The set of parameters to be sought by the inverse problem are electrical conductivities and imaginary parts of relative permittivities since they are the material properties responsible for light-to-heat generation in the source term $\dot{\mathrm{S}}$ of the governing equation. This is achieved by using a minimisation algorithm, which in this work is done by GA.

The GAs are search algorithms based on the mechanics of natural selection and genetics [11]. It simulates the biological mechanism of survival competition and its implementation is shown in Figure 2.

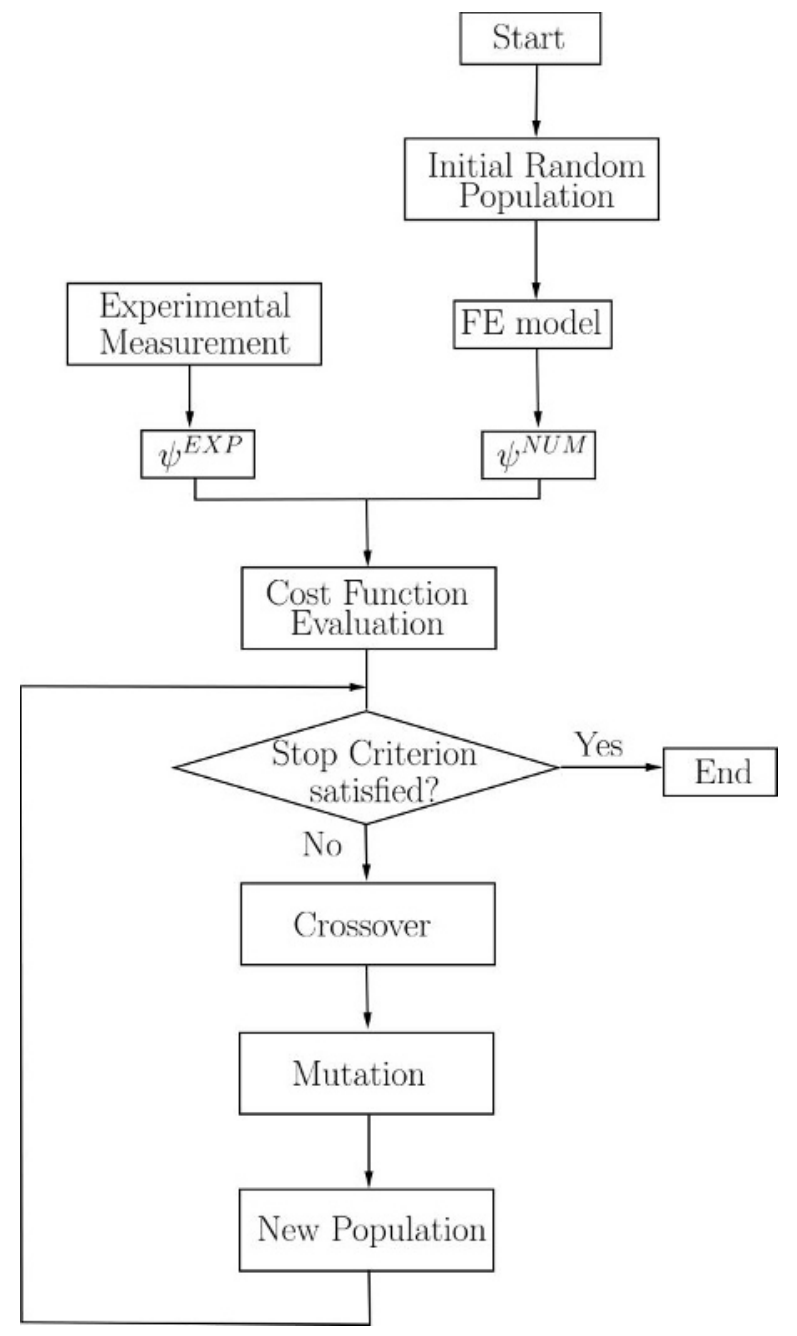

Fig. 2. Flowchart of the inverse problem solution by genetic algorithms.

In GAs, a population of candidate solutions (often denoted as chromosomes) in the problem domain is randomly generated for the initialisation of the algorithm. Solutions for the set of sought parameters are generated iteratively after the evaluation of the CF. Genetic operators like crossover and mutation are defined to produce the new population of 
chromosomes, which share many of the features of their parents. Then, the child chromosomes that better fitness substitute some of their parent choromosomes and the simulation is run until a stopping criterion (for instance the number of generations) is satisfied. Generally, the average fitness of the newly generated populations increases progressively since only the best individuals are selected for breeding the next generations.

The model considered for the present simulations is composed of a single NP surrounded by the liquid medium in which the former is suspended. Figure 3 shows a schematic representation of the domain of study with its boundary conditions (BCs).

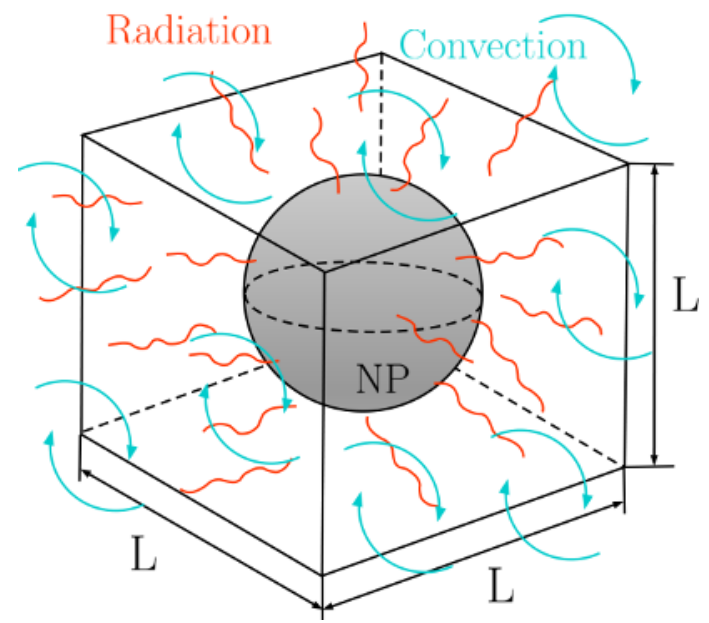

Fig. 3. Schematic representation of the geometry and boundary conditions of a single NP surrounded by a fluid medium in a cube.

With regard to temperature $\mathrm{BCs}$, the domain surfaces are left unconstrained so that the average temperature of the nanofluid can evolve with the dissipation of solar irradiation. Natural convection $(\mathrm{h}=$ $5 \mathrm{~W} /(\mathrm{m} 2 \cdot \mathrm{K}))$ and radiation $(\sigma=1,3360 \bullet 10-9 \mathrm{~W} / \mathrm{K} 4)$ boundary conditions are also considered on the six surfaces of the domain in an attempt to reproduce the experimental conditions at which temperature measurements were performed. For the numerical simulation, the initial temperature is assumed to be the same than for the ambient temperature, $\mathrm{T}_{\mathrm{i}}=25^{\circ} \mathrm{C}$. The bulk estimates of material properties of $\mathrm{Au}$ NPs retained for the simulations of the forward problem can be found in Table 1.

Table 1. Summary of material properties for Au NPs and water.

\begin{tabular}{llll}
\hline Material & Properties & Values & Units \\
\hline \multirow{3}{*}{ Gold $(\mathrm{Au})$} & $\rho$ & 19320 & $\mathrm{~kg} / \mathrm{m}^{3}$ \\
& $\mathrm{\kappa}$ & 131.4 & $\mathrm{~J} /(\mathrm{kg} \cdot \mathrm{K})$ \\
& $\gamma$ & 312 & $\mathrm{~W} /(\mathrm{m} \cdot \mathrm{K})$ \\
& $\rho$ & $3.17 \cdot 10^{7}$ & $(\Omega \cdot \mathrm{m})^{-1}$ \\
\hline \multirow{3}{*}{ Water } & $c$ & 988.037 & $\mathrm{~kg} / \mathrm{m}^{3}$ \\
$\left(\mathrm{H}_{2} \mathrm{O}\right)$ & $\kappa$ & 4176.84 & $\mathrm{~J} /(\mathrm{kg} \cdot \mathrm{K})$ \\
& $\gamma$ & 0.645 & $\mathrm{~W} /(\mathrm{m} \cdot \mathrm{K})$ \\
& & $5.5 \cdot 10^{-6}$ & $(\Omega \cdot \mathrm{m})^{-1}$ \\
\hline
\end{tabular}

Spectral values of relative permittivity of $\mathrm{Au}$ are available in the database Refractiveindex.info [12]. Notice that an spectrum-averaged value of relative permittivity and a dispersion of $10 \%$ around the nominal value of electrical conductivity of $\mathrm{Au}$ reported in Table 1 are considered for numerical simulations.

\section{Results and discussion}

Concerning experimental measurements, the registered values of temperature increase of $\mathrm{Au}$ nanofluids subjected to optical excitation are depicted in Figure 4.

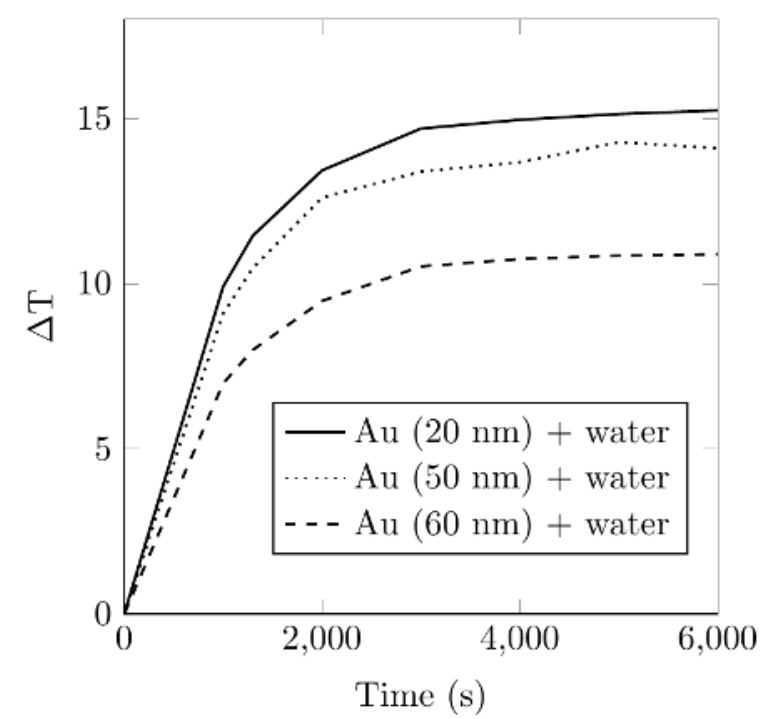

Fig. 4. Evolution of temperature increase for Au nanofluids with different sizes of NPs.

From the numerical simulations and the experimental results reported in Figure 4, the values of the material parameters: relative permittivity and electrical conductivity, are obtained.

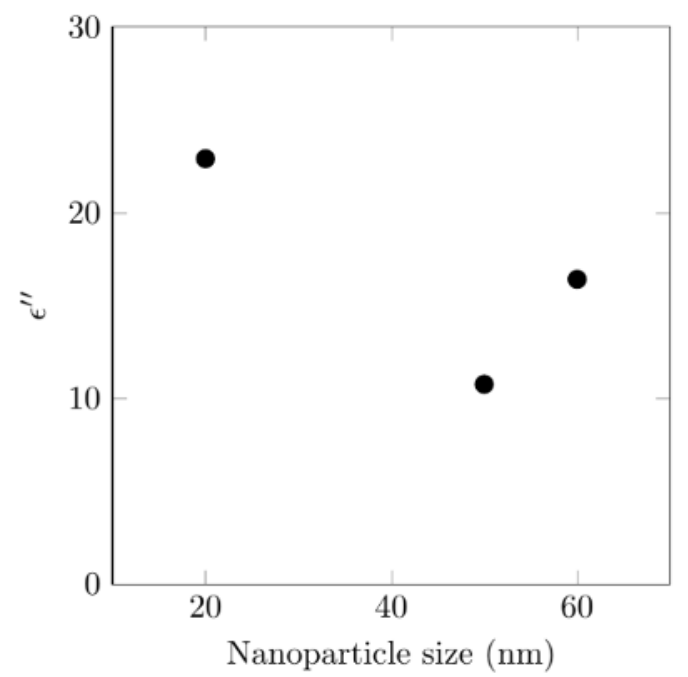

Fig. 5. Imaginary part of relative permittivity for $20 \mathrm{~nm}, 50$ $\mathrm{nm}$ and $60 \mathrm{~nm} \mathrm{Au}$ nanoparticles. 


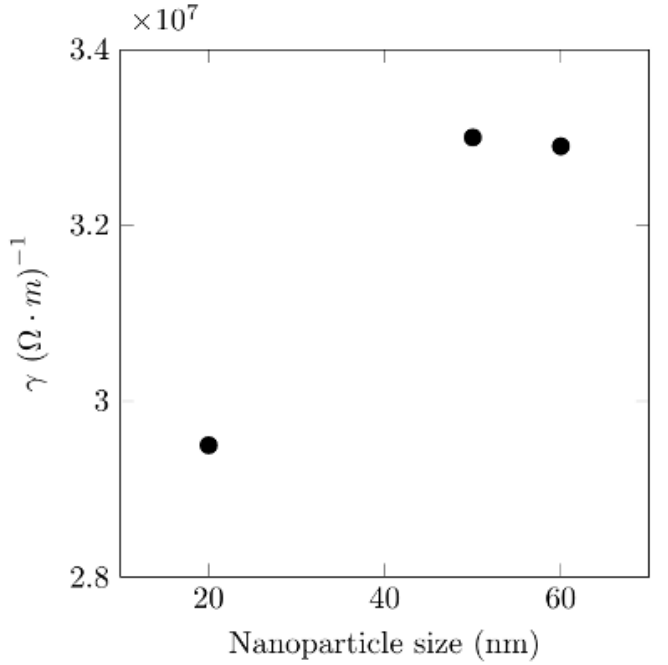

Fig. 6. Electrical conductivity for $20 \mathrm{~nm}, 50 \mathrm{~nm}$ and $60 \mathrm{~nm}$ Au nanoparticles.

Figure 5 represents the data of the imaginary part of relative permittivity with respect to the $\mathrm{Au}$ nanoparticle sizes. The numerical result obtained for this parameter is an averaged value covering the spectrum of relative permmitivity bulk values reported in the literature. It can be observed that predicted values of the imaginary part of relative permittivity are 22.91, 10.76 and 16.41 for $20 \mathrm{~nm}, 50 \mathrm{~nm}$ and $60 \mathrm{~nm}$, respectively, which are within the range of possible experimental values of the imaginary part of relative permittivity of bulk gold: [1.5-27.9], extracted from the database Refractiveindex.info [12].

Figure 6 represents the data of electrical conductivity according to the $\mathrm{Au}$ nanoparticle sizes. The order of magnitude of this physical magnitude falls within the same range than values of bulk electrical conductivity of gold reported in literature, as it can be seen in Table 1.

Therefore, the combination of experimental measurements of temperature increase for different nanoparticle sizes with the present numerical tool can help in the characterisation of the evolution of the physical parameters for different nanoparticle sizes, which still requires more study since a lot of dispersion exists in the reported values of material properties at the nanoscale [12].

a)

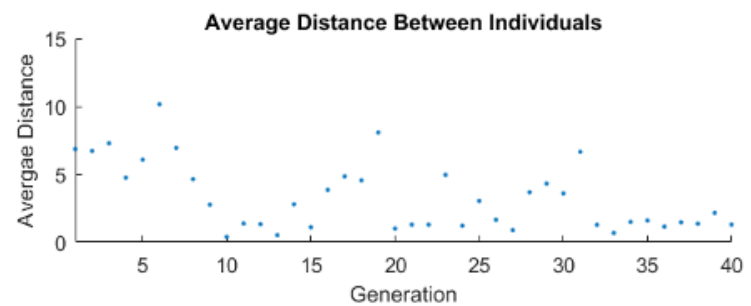

b)

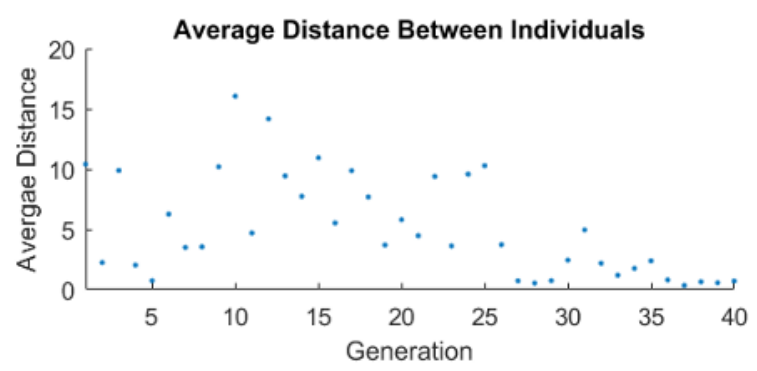

c)

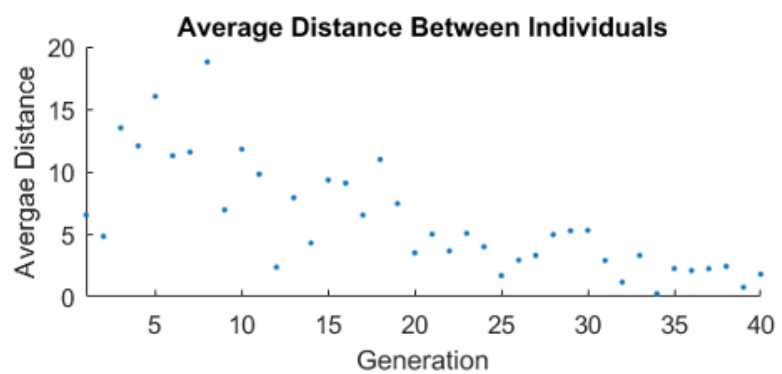

Fig. 7. Evolution of GA convergence for simulations of NPs of a) $20 \mathrm{~nm}$, b) $50 \mathrm{~nm}$ and c) $60 \mathrm{~nm}$ in diameter.

The numerical convergence of GAs for the different nanoparticle sizes simulated in the present work is depicted in Figure 7, where the "Average Distance" represents the diversity in the population of choromosomes. The low values of average distance for the last generations of GAs in Figure 7 show that, at this final stage of the simulation, most of choromosomes possess similar features, which means that the simulation converges towards a numerical solution.

\section{Conclusions}

This work presents a numerical tool with the aim of helping in the characterisation of optical properties of NPs for their application in solar nanofluids. More precisely, imaginary part of relative permittivity and electrical conductivity are the parameters of interest since they govern the light-to-heat conversion. For this purpose, an inverse problem based on the solution technique of genetic has been developed to minimise the cost function of the problem.

From the numerical simulations, the values of the imaginary part of relative permittivity and electrical conductivity is obtained for different sizes of NPs and the obtained results fall within the range of experimental values reported in literature for these bulk properties.

Therefore, this numerical model could be used not only to help in the characterisation of the material properties of NPs from indirect measurements but can also contribute to reduce the number of experiments to be performed for their characterisation. 


\section{Acknowledgements}

This research was partially funded by Ministerio de Economía y Competitividad (MINECO) of Spain through the project ENE2016-77694-R, by Generalitat Valenciana through the project PROMETEU/2020/029 and by the Universitat Jaume I through the project UJI-B2020-32. Josep Forner-Escrig thanks Ministerio de Economía, Industria y Competitividad of Spain and Fondo Social Europeo for a predoctoral fellowship through Grant Ref. BES-2017-080217 (FPI program).

\section{References}

1. International Energy Agency. (IEA), Solar energy perspectives. Paris: International Energy Agency, (2011).

2. R. Bertocchi, J. Karni, A. Kribus, Energy, 29, 687700 (2004).

3. S. Choi and J. Eastman, Enhancing thermal conductivity of fluids with nanoparticles, in Proceedings of the ASME International Mechanical Engineering Congress and Exposition, 12-17, November 1995, San Francisco, CA, USA, (1995).

4. T.P. Otanicar, P. Phelan, J.S. Golden. Sol. Energy 83, 969-77 (2009).

5. T.P. Otanicar, P.E. Phelan, R.S. Prasher, G.

Rosengarten, R. A. Taylor. J. Renew. Sustain. Energy 2, 033102 (2010).

6. A. Zeiny, H. Jin, L. Bai, G. Lin, D. Wen. Sol. Energy, 161, pp.74-82, 2018.

7. J. Forner-Escrig, R. Mondragón, L. Hernández, R. Palma. Int. J. of Mech. Sci., 188, 105952 (2020).

8. A. Gimeno-Furió, R. Martínez-Cuenca, R. Mondragón, A.F. Vela-Gasulla, C. DoñateBuendía, G. Mínguez-Vega, L. Hernández. Energy, 212, 118763 (2020).

9. R. Taylor. FEAP. A finite element analysis program: programmer manual. University of California, Berkeley; 2014.

http://www.ce.berkeley.edu/feap

10. G. Rus, R. Palma, J. L. Pérez-Aparicio. Int. J. Eng. Sci., 47, 554-572 (2009).

11. D. E. Goldberg. Genetic Algorithms in Search, Optimization and Machine Learning. Boston: Addison-Wesley, 1989.

12. M. Polyanskiy. Refractiveindex.info website. https://refractiveindex.info/ Last Accessed: 07-022021 